\title{
173.
}

\section{ON LAPLACE'S METHOD FOR THE ATTRACTION OF ELLIPSOIDS.}

[From the Quarterly Mathematical Journal, vol. I. (1857), pp. 285-300.]

[THE method referred to is that given in Book III. of the Mécanique Céleste, Ed. I. 1798.] Let the equation of the surface of the ellipsoid be

$$
l x^{2}+m y^{2}+n z^{2}-k=0 \text {, }
$$

and take $a, b, c$ as the coordinates of the attracted point, which is supposed to be exterior to the surface. Imagine an indefinitely thin cone, having the attracted point for vertex, and intersecting the surface of the ellipsoid; let $\xi, \eta, \zeta$ be the directioncosines of the cone, and $d S$ its spherical angle. Then if for a moment $r$ denote the radius vector corresponding to a point within the ellipsoid, the element of the mass within the cone is $r^{2} d r d S$; and we may thence, by an integration with respect to $r$, find the attractions parallel to the axes (and tending towards the centre) and the potential of the mass within the cone, viz. if $r^{\prime}, r^{\prime \prime}$ be the values of $r$ at the surface of the ellipsoid, the attractions are $\left(r^{\prime \prime}-r^{\prime}\right) \xi d S,\left(r^{\prime \prime}-r^{\prime}\right) \eta d S,\left(r^{\prime \prime}-r^{\prime}\right) \xi d S$, and the potential is $\frac{1}{2}\left(r^{\prime \prime 2}-r^{\prime 2}\right) d S$. And putting for shortness

$$
\begin{aligned}
& L=l \xi^{2}+m \eta^{2}+n \zeta^{2}, \\
& I=l a \xi+m b \eta+n c \zeta, \\
& P=l a^{2}+m b^{2}+n c^{2}-1, \\
& R=I^{2}-P L,
\end{aligned}
$$

then $r^{\prime}, r^{\prime \prime}$ will be the roots of the equation $L r^{2}-2 I r+P=0$, and we have consequently $r^{\prime}=\frac{I-\sqrt{ } R}{L}, r^{\prime \prime}=\frac{I+\sqrt{ } R}{L}$. We have, therefore, for the attractions parallel to the axes 
(and tending towards the centre) and for the potential of the entire ellipsoid the well-known expressions

$$
\begin{aligned}
& A=2 \int d S \frac{\xi \sqrt{ } R}{L}, \\
& B=2 \int d S \frac{\eta \sqrt{ } R}{L}, \\
& C=2 \int d S \frac{\zeta \sqrt{ } R}{L},
\end{aligned}
$$

and

$$
V=2 \int \frac{I \sqrt{ } R d S}{L^{2}}
$$

where the integrations extend over the spherical angle of the circumscribed cone $R=0$.

We have moreover, by the general theory of attractions,

$$
A=-\frac{d V}{d a}, B=-\frac{d V}{d b}, C=-\frac{d V}{d c} .
$$

Since at the limits of the integration the quantity under the integral sign vanishes, it is easy to see that the first differentials of $V, A, B, C$ with respect to any of the quantities $a, b, c, l, m, n, k$, may be found by simply differentiating under the integral sign without its being necessary to pay attention to the variation of the limits, so. that, for instance, $\frac{d V}{d a}=2 \int d S \frac{d}{d a} \frac{I \sqrt{ } R}{L}$. It is proper to remark that the expressions thus obtained for the attractions $A, B, C$, are of a different form from the foregoing expressions for the same quantities. Laplace writes

$$
F=a A+b B+c C,
$$

and he remarks that it may be shown by differentiation that the quantities, $B, C, F, V$, are connected by an equation which (writing $k$ for $k^{2}$, and $\frac{m}{l}, \frac{n}{l}$ for $m, n$, the equation of the ellipsoid being with Laplace $x^{2}+m y^{2}+n z^{2}=k^{2}$ ) becomes, in the notation of the present memoir,

$$
\begin{gathered}
\left\{l\left(a^{2}+b^{2}+c^{2}\right)-k\right\}\left(\frac{d V}{d k}-\frac{d F}{d k}\right)+V-F \\
+\left(1-\frac{l}{m}\right) b\left(\frac{d F}{d b}-\frac{1}{2} \frac{d V}{d b}-B\right) \\
+\left(1-\frac{l}{n}\right) c\left(\frac{d F}{d c}-\frac{1}{2} \frac{d V}{d c}-C\right) \\
-(m-l) \frac{d F}{d m}-(n-l) \frac{d F}{d n}=0 .
\end{gathered}
$$


I write this equation in the form

$$
\begin{aligned}
\left(l a^{2}+\right. & \left.m b^{2}+n c^{2}-k\right)\left(\frac{d V}{d k}-\frac{d F}{d k}\right)+V-F \\
& +(l-l)\left\{-a^{2}\left(\frac{d V}{d k}-\frac{d F}{d k}\right)+\frac{a}{l}\left(\frac{d F}{d a}-\frac{1}{2} \frac{d V}{d a}-A\right)-\frac{d F^{\prime}}{d l}\right\} \\
& +(m-l)\left\{-b^{2}\left(\frac{d V}{d k}-\frac{d F}{d k}\right)+\frac{b}{m}\left(\frac{d F}{d b}-\frac{1}{2} \frac{d V}{d b}-B\right)-\frac{d F}{d m}\right\} \\
& +(n-l)\left\{-c^{2}\left(\frac{d V}{d k}-\frac{d F}{d k}\right)+\frac{c}{n}\left(\frac{d F}{d c}-\frac{1}{2} \frac{d V}{d c}-C\right)-\frac{d F}{d n}\right\} \\
& =0
\end{aligned}
$$

and I remark that this equation may be broken up into two equations, each of which separately is satisfied; these equations are

$$
\begin{aligned}
-k\left(\frac{d V}{d k}-\frac{d F}{d k}\right)+(V & \left.-F^{\prime}\right)-l \frac{d F}{d l}-m \frac{d F}{d m}-n \frac{d F}{d n} \\
& +a\left(\frac{d F}{d a}-\frac{1}{2} \frac{d V}{d a}-A\right)+b\left(\frac{d F}{d b}-\frac{1}{2} \frac{d V}{d b}-B\right)+c\left(\frac{d F}{d c}-\frac{1}{2} \frac{d V}{d c}-C\right)=0
\end{aligned}
$$

and

$$
\begin{aligned}
\left(a^{2}+b^{2}+c^{2}\right)\left(\frac{d V}{d k}\right. & \left.-\frac{d F}{d k}\right)-\frac{d F}{d l}-\frac{d F}{d m}-\frac{d F}{d n} \\
& +\frac{a}{l}\left(\frac{d F}{d a}-\frac{1}{2} \frac{d V}{d a}-A\right)+\frac{b}{m}\left(\frac{d F}{d b}-\frac{1}{2} \frac{d V}{d b}-B\right)+\frac{c}{n}\left(\frac{d F}{d c}-\frac{1}{2} \frac{d V}{d c}-C\right)=0 .
\end{aligned}
$$

It may be added that the functions under the integral signs, and consequently the integrals, are all of them homogeneous of the degree zero in $l, m, n, k$. The thing to be verified is that the foregoing two equations are satisfied by the functions under the integral signs, independently of the integrations, in fact by the values

$$
\left\{\begin{array}{l}
A=\frac{\xi \sqrt{ } R}{L}, B=\frac{\eta \sqrt{ } R}{L}, C=\frac{\zeta \sqrt{ } R}{L}, \\
V=\frac{I \sqrt{ } R}{L} \\
F=\frac{(a \xi+b \eta+c \zeta) \sqrt{ } R}{L} .
\end{array}\right.
$$

We find by differentiating these values, and after a few obvious reductions,

$$
\begin{aligned}
& \frac{d V}{d a}=l \xi\left(\frac{\sqrt{ } R}{L^{2}}+\frac{I^{2}}{L^{2} \sqrt{ } R}\right)+l a\left(\frac{-I}{L \sqrt{ } R}\right), \\
& \frac{d V}{d l}=a \xi\left(\frac{\sqrt{ } R}{L^{2}}+\frac{I^{2}}{L^{2} \sqrt{ } R}\right)+a^{2}\left(\frac{-I}{2 L \sqrt{ } R}\right)+\xi^{2}\left(-\frac{3 I \sqrt{ } R}{2 L^{3}}-\frac{I^{3}}{2 L^{3} \sqrt{ } R}\right), \\
& \frac{d V}{d k}= \\
& \frac{I}{2 L \sqrt{ } R},
\end{aligned}
$$




$$
\begin{array}{ll}
\frac{d F}{d a}-\frac{\xi \sqrt{ } R}{L} & =(a \xi+b \eta+c \zeta)\left\{l \zeta\left(\frac{I}{L \sqrt{ } R}\right)+l a\left(-\frac{1}{\sqrt{ } R}\right)\right. \\
\frac{d F}{d l} & =(a \xi+b \eta+c \zeta)\left\{a \xi\left(\frac{I}{L \sqrt{ } R}\right)+a^{2}\left(\frac{-1}{2 \sqrt{ } R}\right)+\xi^{2}\left(-\frac{\sqrt{ } R}{2 L^{2}}-\frac{I^{2}}{2 L^{2} \sqrt{ } R}\right)\right\}, \\
\frac{d F}{d k} & =(a \xi+b \eta+c \zeta)\{
\end{array}
$$

values which give, as they should do,

$$
l \frac{d F}{d l}+m \frac{d F}{d m}+n \frac{d F}{d n}+k \frac{d F}{d k}=0
$$

Hence forming the values of the different parts of the first equation,

$$
\begin{aligned}
& -k\left(\frac{d V}{d k}-\frac{d F}{d k}\right)+V-F-l \frac{d F}{d l}-m \frac{d F}{d m}-n \frac{d F}{d n} \\
& =\frac{I \sqrt{ } R}{L^{2}}-\frac{k I}{2 L \sqrt{ } R}+(a \xi+b \eta+c \zeta)\left\{-\frac{\sqrt{ } R}{L}+\frac{k}{\sqrt{ } R}\right\}, \\
& a\left(\frac{d F}{d a}-A\right)+b\left(\frac{d F}{d b}-B\right)+c\left(\frac{d F}{d c}-C\right)=(a \xi+b \eta+c \zeta)\left\{\frac{\sqrt{ } R}{L}-\frac{k}{\sqrt{ } R}\right\}, \\
& -\frac{1}{2}\left(a \frac{d V}{d a}+b \frac{d V}{d b}+c \frac{d V}{d c}\right)=\quad-\frac{I \sqrt{ } R}{L^{2}}+\frac{k I}{2 L \sqrt{ } R},
\end{aligned}
$$

which satisfy the first equation.

And in like manner for the second equation,

$$
\begin{gathered}
-\left(a^{2}+b^{2}+c^{2}\right)\left(\frac{d V}{d k}-\frac{d F}{d k}\right)=-\left(a^{2}+b^{2}+c^{2}\right) \frac{I}{2 L \sqrt{ } R}+\left(a^{2}+b^{2}+c^{2}\right)(a \xi+b \eta+c \zeta) \frac{1}{2 \sqrt{ } R} \\
\begin{array}{c}
\frac{a}{l}\left(\frac{d F}{d a}-A\right)+\frac{b}{m}\left(\frac{d F}{d b}-B\right)+\frac{c}{n}\left(\frac{d F}{d c}-C\right) \\
=(a \xi+b \eta+c \zeta)^{2} \frac{I}{L \sqrt{ } R}-\left(a^{2}+b^{2}+c^{2}\right)(a \xi+b \eta+c \zeta) \frac{1}{\sqrt{ } R} \\
-\frac{1}{2}\left(\frac{a}{l} \frac{d V}{d a}+\frac{b}{m} \frac{d V}{d b}+\frac{c}{n} \frac{d V}{d c}\right)=\left(a^{2}+b^{2}+c^{2}\right) \frac{I}{2 L \sqrt{ } R}-(a \xi+b \eta+c \zeta)\left(\frac{\sqrt{ } R}{2 L^{2}}+\frac{I^{2}}{2 L^{2} \sqrt{ } R}\right) \\
-\frac{d F}{d l}-\frac{d F}{d m}-\frac{d F}{d n}=-(a \xi+b \eta+c \zeta)^{2} \frac{I}{2 L \sqrt{ } R}+\left(a^{2}+b^{2}+c^{2}\right)(a \xi+b \eta+c \zeta) \frac{1}{2 \sqrt{ } R} \\
+(a \xi+b \eta+c \zeta)\left(\frac{\sqrt{ } R}{2 L^{2}}+\frac{I^{2}}{2 L^{2} \sqrt{ } R}\right)
\end{array}
\end{gathered}
$$

which satisfy the second equation 
Now considering $V, F, A, B, C$ as standing for the definite integrals, we may replace $A, B, C$ by the differential coefficients of $V$, and retaining for shortness $F$ to stand for

$$
F=-a \frac{d V}{d a}-b \frac{d V}{d b}-c \frac{d V}{d c}
$$

the first equation becomes

$$
\begin{aligned}
-k\left(\frac{d V}{d k}-\frac{d F}{d k}\right)+V & -F-l \frac{d F}{d l}-m \frac{d F}{d m}-n \frac{d F}{d n} \\
& +a\left(\frac{d F}{d a}+\frac{1}{2} \frac{d V}{d a}\right)+b\left(\frac{d F}{d b}+\frac{1}{2} \frac{d V}{d b}\right)+c\left(\frac{d F}{d c}+\frac{1}{2} \frac{d V}{d c}\right)=0
\end{aligned}
$$

and the second equation becomes

$$
\begin{aligned}
-\left(a^{2}+b^{2}+c^{2}\right)\left(\frac{d V}{d k}-\frac{d F}{d k}\right) & -\frac{d F}{d l}-\frac{d F}{d m}-\frac{d F}{d n} \\
& +\frac{a}{l}\left(\frac{d F}{d a}+\frac{1}{2} \frac{d V}{d a}\right)+\frac{b}{m}\left(\frac{d F}{d b}+\frac{1}{2} \frac{d V}{d b}\right)+\frac{c}{n}\left(\frac{d F}{d c}+\frac{1}{2} \frac{d V}{d c}\right)=0 .
\end{aligned}
$$

If we put as before $V=\frac{I \sqrt{ } R}{L^{2}}$, the preceding values of the differential coefficients of $V$ give $F=\frac{2 I \sqrt{ } R}{L^{2}}+\frac{k I}{L \sqrt{ } R}$, or as we may write it $F=-2 V+W$, where $W=\frac{k I}{L \sqrt{ } R}$. I put then for the moment

$$
\left\{\begin{array}{l}
V=\frac{I \sqrt{ } R}{L^{2}}, W=\frac{k I}{L \sqrt{ } R} \\
F=-2 V+W .
\end{array}\right.
$$

It should be remarked that there is nothing in what has preceded which tends to show that these values must satisfy the differential equations. The definite integrals must, of course, satisfy as before the equations, but it does not follow that the equations are satisfied by the elements separately. And in fact only the first equation is so satisfied; the second equation is not satisfied. To verify this I form the differential coefficients

$$
\begin{aligned}
& \frac{d W}{d a}=l \xi\left(\frac{k}{L \sqrt{ } R}-\frac{k I^{2}}{L R \sqrt{ } R}\right)+l a\left(\frac{k I}{R \sqrt{ } R}\right) \\
& \frac{d W}{d k}=\frac{I}{L \sqrt{ } R}-\frac{k I}{2 R \sqrt{ } R}, \\
& \frac{d W}{d l}=a \xi\left(\frac{k}{L \sqrt{ } R}-\frac{k I^{2}}{L R \sqrt{ } R}\right)+\xi^{2}\left(\frac{-3 k I}{2 L^{2} \sqrt{ } R}+\frac{k I^{3}}{2 L^{2} R \sqrt{ } R}\right)+a^{2} \frac{k I}{2 R \sqrt{ } R} .
\end{aligned}
$$

C. III. 
We have as before

$$
l \frac{d F}{d l}+m \frac{d F}{d m}+n \frac{d F}{d n}+k \frac{d F}{d k}=0
$$

and forming the expressions for the different parts of the first equation,

$$
\begin{aligned}
& -k\left(\frac{d V}{d k}-\frac{d F}{d k}\right)+V-F-l \frac{d F}{d l}-m \frac{d F}{d m}-n \frac{d F}{d n} \\
& =-5 k \frac{d V}{d k}+2 k \frac{d W}{d k}+3 V-W \\
& =\frac{3 I \sqrt{ } R}{L^{2}}-\frac{3 k I}{2 L \sqrt{ } R}-\frac{k^{2} I}{R \sqrt{ } R}, \\
& a\left(\frac{d F}{d a}+\frac{1}{2} \frac{d V}{d a}\right)+\& c .= \\
& -\frac{3}{2}\left(a \frac{d V}{d a}+b \frac{d V}{d b}+c \frac{d V}{d c}\right)+\left(a \frac{d W}{d a}+b \frac{d W}{d b}+c \frac{d W}{d c}\right),
\end{aligned}
$$

and

$$
\begin{array}{rlrl}
-\frac{3}{2}\left(a \frac{d V}{d a}+b \frac{d V}{d b}+c \frac{d V}{d c}\right) & =-\frac{3 I \sqrt{ } R}{L^{2}}+\frac{3 k I}{2 L \sqrt{ } R}, \\
\left(a \frac{d W}{d a}+b \frac{d W}{d b}+c \frac{d W}{d c}\right) & = & \frac{k^{2} I}{R \sqrt{ } R},
\end{array}
$$

which show that the first equation is satisfied.

Proceeding in the same manner with the second equation,

$$
\begin{aligned}
& -\left(a^{2}+b^{2}+c^{2}\right)\left(\frac{d V}{d k}-\frac{d F}{d k}\right)= \\
& -\left(a^{2}+b^{2}+c^{2}\right)\left(3 \frac{d V}{d k}-\frac{d W}{d k}\right)=\left(a^{2}+b^{2}+c^{2}\right)\left(-\frac{I}{L \sqrt{ } R}-\frac{k I}{2 R \sqrt{ } R}\right) \\
& \quad \frac{a}{l}\left(\frac{d F}{d a}+\frac{1}{2} \frac{d V}{d a}\right)+\& c .= \\
& -\frac{3}{2}\left(\frac{a}{l} \frac{d V}{d a}+\frac{b}{m} \frac{d V}{d b}+\frac{c}{n} \frac{d V}{d c}\right)+\left(\frac{a}{l} \frac{d W}{d a}+\frac{b}{m} \frac{d W}{d b}+\frac{c}{n} \frac{d W}{d c}\right) ;
\end{aligned}
$$

and

$$
\begin{aligned}
& -\frac{3}{2}\left(\frac{a}{l} \frac{d V}{d a}+\frac{b}{m} \frac{d V}{d b}+\frac{c}{n} \frac{d V}{d c}\right)=\left(a^{2}+b^{2}+c^{2}\right) \frac{3 I}{2 L \sqrt{ } R}+(a \xi+b \eta+c \zeta)\left(-\frac{3 \sqrt{ } R}{2 L^{2}}-\frac{3 I^{2}}{2 L^{2} \sqrt{ } R}\right) \\
& \left(\frac{a}{l} \frac{d W}{d a}+\frac{b}{m} \frac{d W}{d b}+\frac{c}{n} \frac{d W}{d c}\right)=\left(a^{2}+b^{2}+c^{2}\right) \frac{k I}{R \sqrt{ } R}+(a \xi+b \eta+c \zeta)\left(\frac{k}{L \sqrt{ } R}-\frac{k I^{2}}{L R \sqrt{ } R}\right) \\
& -\frac{d F}{d l}-\frac{d F}{d m}-\frac{d F}{d n}=2\left(\frac{d V}{d l}+\frac{d V}{d m}+\frac{d V}{d n}\right)-\left(\frac{d W}{d l}+\frac{d W}{d m}+\frac{d W}{d n}\right)
\end{aligned}
$$


and

$$
\begin{array}{r}
2\left(\frac{d V}{d l}+\frac{d V}{d m}+\frac{d V}{d n}\right)=\left(a^{2}+b^{2}+c^{2}\right)\left(\frac{-1}{L \sqrt{ } R}\right)+(a \xi+b \eta+c \zeta)\left(\frac{2 \sqrt{ } R}{L^{2}}+\frac{2 I^{2}}{L^{2} \sqrt{ } R}\right)-\frac{3 I \sqrt{ } R}{L^{3}}-\frac{I^{3}}{L^{3} \sqrt{ } R} \\
-\left(\frac{d W}{d l}+\frac{d W}{d m}+\frac{d W}{d n}\right)=\left(a^{2}+b^{2}+c^{2}\right)\left(\frac{-k I}{2 R \sqrt{ } R}\right)+(a \xi+b \eta+c \zeta)\left(\frac{-k}{L \sqrt{ } R}+\frac{k I^{2}}{L R \sqrt{ } R}\right) \\
+\frac{3 k I}{2 L^{2} \sqrt{ } R}-\frac{k I^{3}}{2 L^{2} R \sqrt{ } R}
\end{array}
$$

and the value of the left-hand side of the second equation is therefore,

$$
(a \xi+b \eta+c \zeta)\left(\frac{\sqrt{ } R}{2 L^{2}}+\frac{3 I^{2}}{2 L^{2} \sqrt{ } R}\right)-\frac{3 I \sqrt{ } R}{L^{3}}-\frac{I^{3}}{2 L^{3} \sqrt{ } R}+\frac{3 k I}{2 L^{2} \sqrt{ } R}-\frac{k I^{3}}{2 L^{2} R \sqrt{ } R},
$$

which is not equal to zero, or the second equation is not satisfied.

I consider again $V, F$ as denoting the definite integrals, and I eliminate $\frac{d V}{d k}, \frac{d F^{r}}{d k}$ by means of the equations

$$
\begin{aligned}
& l \frac{d V}{d l}+m \frac{d V}{d m}+n \frac{d V}{d n}+k \frac{d V}{d k}=0 \\
& l \frac{d F}{d l}+m \frac{d F}{d m}+n \frac{d F}{d n}+k \frac{d F}{d k}=0
\end{aligned}
$$

The first equation thus becomes

$$
\begin{aligned}
l \frac{d V}{d l}+m \frac{d V}{d m}+n \frac{d V}{d n}- & 2\left(l \frac{d F}{d l}+m \frac{d F}{d m}+n \frac{d F}{d n}\right)+V-F \\
& +\frac{1}{2}\left(a \frac{d V}{d a}+b \frac{d V}{d b}+b \frac{d V}{d c}\right)+\left(a \frac{d F}{d a}+b \frac{d F}{d b}+c \frac{d F}{d c}\right)=0
\end{aligned}
$$

and the second equation becomes

$$
\begin{aligned}
\left(a^{2}+\right. & \left.b^{2}+c^{2}\right) \frac{1}{k}\left(l \frac{d V}{d l}+m \frac{d V}{d m}+n \frac{d V}{d n}\right)-\left(a^{2}+b^{2}+c^{2}\right) \frac{1}{k}\left(l \frac{d F}{d l}+m \frac{d F}{d m}+n \frac{d F}{d n}\right) \\
& +\frac{1}{2}\left(\frac{a}{l} \frac{d V}{d a}+\frac{b d}{m} \frac{V}{d b}+\frac{c}{n} \frac{d V}{d c}\right)+\left(\frac{a}{l} \frac{d F}{d a}+\frac{b}{m} \frac{d F}{d b}+\frac{c}{n} \frac{d F}{d c}\right)-\left(\frac{d F}{d l}+\frac{d F}{d m}+\frac{d F}{d n}\right)=0
\end{aligned}
$$

and it will be remembered that in these equations

$$
F=-a \frac{d V}{d a}-b \frac{d V}{d b}-c \frac{d V}{d c}
$$

The first equation (it is easy to perceive) shows merely that $V$ is made up of terms separately homogeneous in $a, b, c$, and in $l, m, n$, and such that the degrees in the two sets respectively being $\kappa, \lambda$, then $\lambda-\frac{1}{2}(\kappa-2)$. In fact $V$ being a function of the form in question, if we attend only to the term the degrees of which are $\kappa, \lambda$, 
then, by the properties of homogeneous functions, $F=-V$, and the first equation is satisfied if only

$$
\lambda+2 \lambda \kappa+1+\kappa+\frac{1}{2} \kappa-\kappa^{2}=0,
$$

i.e. if $\lambda(2 \kappa+1)=\kappa^{2}-\frac{3}{2} \kappa-1=\frac{1}{2}(\kappa-2)(2 \kappa+1)$ or $\lambda=\frac{1}{2}(\kappa-2)$. Or, what is the same thing, we may say that the first equation shows that $V$ is made up of terms the degrees of which in $a, b, c$ and in $l, m, n$ are $-2 i-1$, and $-i-\frac{3}{2}$ respectively. Attending henceforth only to the second equation, I write $\frac{l}{k}=\frac{1}{\alpha+\theta}, \frac{m}{k}=\frac{1}{\beta+\theta}, \frac{n}{k}=\frac{1}{\gamma+\theta}$; so that $\alpha+\theta, \beta+\theta, \gamma+\theta$ denote the squared semiaxes of the ellipsoid. We have

$$
\frac{d}{d l}=-\frac{(\alpha+\theta)^{2}}{k} \frac{d}{d a}, \& c .
$$

and the equation becomes

$$
\begin{array}{r}
-\left(a^{2}+b^{2}+c^{2}\right)\left((\alpha+\theta) \frac{d V}{d \alpha}+(\beta+\theta) \frac{d V}{d \beta}+(\gamma+\theta) \frac{d V}{d \gamma}\right) \\
+\left(a^{2}+b^{2}+c^{2}\right)\left((\alpha+\theta) \frac{d F}{d \alpha}+(\beta+\theta) \frac{d F}{d \beta}+(\gamma+\theta) \frac{d F}{d \gamma}\right) \\
+\frac{1}{2}\left(a(\alpha+\theta) \frac{d V}{d a}+b(\beta+\theta) \frac{d V}{d b}+c(\gamma+\theta) \frac{d V}{d c}\right) \\
+\left(a(\alpha+\theta) \frac{d F}{d a}+b(\beta+\theta) \frac{d F}{d b}+c(\gamma+\theta) \frac{d F}{d c}\right) \\
+\left((a+\theta)^{2} \frac{d F}{d \alpha}+(\beta+\theta)^{2} \frac{d F}{d \beta}+(\gamma+\theta)^{2} \frac{d F}{d \gamma}\right)=0 .
\end{array}
$$

Put for shortness $\Theta=(\alpha+\theta)(\beta+\theta)(\gamma+\theta)$, and write

$$
V=v \sqrt{ } \Theta, F=f \sqrt{ } \Theta,
$$

( $\sqrt{ } \Theta$ is to a constant factor près the mass of the ellipsoid) then $v, f$ are connected by the equation

$$
f=-a \frac{d v}{d a}-b \frac{d v}{d b}-c \frac{d v}{d c}
$$

and observing that

$$
\begin{aligned}
& (\alpha+\theta) \frac{d \sqrt{ } \Theta}{d \alpha}+(\beta+\theta) \frac{d \sqrt{ } \Theta}{d \beta}+(\gamma+\theta) \frac{d \sqrt{ } \Theta}{d \gamma}=3 \Theta \frac{1}{2 \sqrt{ } \Theta}=\frac{3}{2} \sqrt{ } \Theta \\
& (\alpha+\theta)^{2} \frac{d \sqrt{ } \Theta}{d \alpha}+(\beta+\theta)^{2} \frac{d \sqrt{ } \Theta}{d \beta}+(\gamma+\theta)^{2} \frac{d \sqrt{ } \Theta}{d \gamma} \\
& =(\alpha+\beta+\gamma+3 \theta) \Theta \frac{1}{2 \sqrt{ } \Theta}=\frac{1}{2}(\alpha+\beta+\gamma+3 \theta) \sqrt{ } \theta
\end{aligned}
$$


the equation becomes

$$
\begin{aligned}
& -\left(a^{2}+b^{2}+c^{2}\right)\left((\alpha+\theta) \frac{d v}{d \alpha}+(\beta+\theta) \frac{d v}{d \beta}+(\gamma+\theta) \frac{d v}{d \gamma}\right) \\
& +\left(a^{2}+b^{2}+c^{2}\right)\left((\alpha+\theta) \frac{d f}{d \alpha}+(\beta+\theta) \frac{d f}{d \beta}+(\gamma+\theta) \frac{d f}{d \gamma}\right) \\
& -\frac{3}{2}\left(a^{2}+b^{2}+c^{2}\right) v+\frac{3}{2}\left(a^{2}+b^{2}+c^{2}\right) f \\
& +\frac{1}{2}\left(a(\alpha+\theta) \frac{d v}{d \alpha}+b(\beta+\theta) \frac{d v}{d b}+c(\gamma+\theta) \frac{d v}{d c}\right) \\
& +\left(a(\alpha+\theta) \frac{d f}{d a}+b(\beta+\theta) \frac{d f}{d b}+c(\gamma+\theta) \frac{d f}{d c}\right) \\
& +\left((\alpha+\theta)^{2} \frac{d f}{d \alpha}+(\beta+\theta)^{2} \frac{d f}{d \beta}+(\gamma+\theta)^{2} \frac{d f}{d \gamma}\right) \\
& +\frac{1}{2}(\alpha+\beta+\gamma+3 \theta) f=0 .
\end{aligned}
$$

Now $v\left(=\frac{1}{\sqrt{ } \Theta} V\right)$ may be expanded in the form

$$
v=U_{0}+U_{1}+U_{2} \ldots+U_{i} \ldots
$$

where $U_{i}$ is of the degree $2 i$ in the semiaxes $\sqrt{ }(\alpha+\theta), \sqrt{ }(\beta+\theta), \sqrt{ }(\gamma+\theta)$, and of the degree $-2 i-1$ in the coordinates $a, b, c$. And it is easy to see that the first term of the expansion is

$$
U_{0}=\frac{4 \pi}{3} \frac{1}{\sqrt{\left(a^{2}+b^{2}+c^{2}\right)}}
$$

The preceding value of $v$ gives

$$
f=U_{0}+3 U_{1}+5 U_{2} \ldots+(2 i+1) U_{i}+\ldots
$$

and substituting the values of $v, f$ in the differential equation, and attending only to the terms which are of the same dimensions as $\left(a^{2}+b^{2}+c^{2}\right) U_{i+1}$, we have

$$
\begin{aligned}
& \left\{-(i+1)+(i+1)(2 i+3)-\frac{3}{2}+\frac{3}{2}(2 i+3)\right\}\left(a^{2}+b^{2}+c^{2}\right) U_{i+1} \\
+ & \left\{a \alpha \frac{d U_{i}}{d \alpha}+b \beta \frac{d U_{i}}{d \beta}+c \gamma \frac{d U_{i}}{d \gamma}-(2 i+1) \theta U_{i}\right\}\left(\frac{1}{2}+2 i+1\right) \\
+ & \left\{\alpha^{2} \frac{d U_{i}}{d \alpha}+\beta^{2} \frac{d U_{i}}{d \beta}+\gamma^{2} \frac{d U_{i}}{d \gamma}+2 i \theta U_{i}-\theta^{2}\left(\frac{d U_{i}}{d \alpha}+\frac{d U_{i}}{d \beta}+\frac{d U_{i}}{d \gamma}\right)\right\}(2 i+1) \\
+ & \frac{1}{2}(\alpha+\beta+\gamma+3 \theta)(2 i+1) U_{i}=0
\end{aligned}
$$


or, reducing,

$$
\begin{aligned}
& (i+1)(2 i+5)\left(a^{2}+b^{2}+c^{2}\right) U_{i+1} \\
+ & \left(2 i+\frac{3}{2}\right)\left(a \alpha \frac{d U_{i}}{d a}+b \beta \frac{d U_{i}}{d b}+c \gamma \frac{d U_{i}}{d c}\right) \\
+ & (2 i+1)\left(\alpha^{2} \frac{d U_{i}}{d \alpha}+\beta^{2} \frac{d U_{i}}{d \beta}+\gamma^{2} \frac{d U_{i}}{d \gamma}+\frac{1}{2}(\alpha+\beta+\gamma) U_{i}\right) \\
- & (2 i+1)\left(\frac{d U_{i}}{d \alpha}+\frac{d U_{i}}{d \beta}+\frac{d U_{i}}{d \gamma}\right)=0 .
\end{aligned}
$$

Now $U_{i}$ is a function of $\alpha+\theta, \beta+\theta, \gamma+\theta$; if, therefore, for any particular value of $i, U_{i}$ is independent of $\theta$, it is clear that $U_{i}$ must be a function of the differences of these quantities, and we shall have $\frac{d U_{i}}{d \alpha}+\frac{d U_{i}}{d \beta}+\frac{d U_{i}}{d \gamma}=0$; and this being so, the equation of differences, and consequently $U_{i+1}$, will be independent of $\theta$. But $U_{0}$ is independent of $\theta$, hence $U_{1}, U_{2}$, \&c. are all of them independent of $\theta$, or $v$ is independent of $\theta$; i.e. for ellipsoids having the same foci for their principal sections, and acting on the same external point, the potentials, and therefore the attractions, are proportional to the masses, which is Maclaurin's theorem for the attractions of ellipsoids upon an external point.

The foregoing equation, omitting the term which vanishes, gives

$$
\begin{gathered}
-\left(2 i+\frac{3}{2}\right)\left(a \alpha \frac{d U_{i}}{d a}+b \beta \frac{d U_{i}}{d b}+c \gamma \frac{d U_{i}}{d c}\right) \\
U_{i+1}=\frac{-(2 i+1)\left(\alpha^{2} \frac{d U_{i}}{d \alpha}+\beta^{2} \frac{d U_{i}}{d \beta}+\gamma^{2} \frac{d U_{i}}{d \gamma}+\frac{1}{2}(\alpha+\beta+\gamma) U_{i}\right)}{(i+1)(2 i+5)\left(a^{2}+b^{2}+c^{2}\right)} .
\end{gathered}
$$

It may be remarked that this equation, with the assistance of the equation

$$
\frac{d U_{i}}{d \alpha}+\frac{d U_{i}}{d \beta}+\frac{d U_{i}}{d \gamma}=0
$$

gives

$$
\begin{aligned}
& (i+1)(2 i+5)\left(\frac{d U_{i+1}}{d \alpha}+\frac{d U_{i+1}}{d \beta}+\frac{d U_{i+1}}{d \gamma}\right)=-\left(2 i+\frac{3}{2}\right)\left(a \frac{d U_{i}}{d a}+b \frac{d U_{i}}{d b}+c \frac{d U_{i}}{d c}\right) \\
& -(2 i+1)\left(2\left(\alpha \frac{d U_{i}}{d \alpha}+\beta \frac{d U_{i}}{d \beta}+\gamma \frac{d U_{i}}{d \gamma}\right)+\frac{3}{2} U_{i}\right)=\left(\left(2 i+\frac{3}{2}\right)(2 i+1)-(2 i+1)\left(2 i+\frac{3}{2}\right)\right) U_{i}=0
\end{aligned}
$$

which is as it should be.

Write

$$
U_{i}=\frac{Q_{i}}{\left(a^{2}+b^{2}+c^{2}\right)^{2 i+\frac{1}{2}}}
$$


where $U_{i}$ is of the degree $i$ in $\alpha, \beta, \gamma$, and of the degree $2 i$ in $a, b, c$. We have

$$
\begin{aligned}
& a \alpha \frac{d U_{i}}{d a}+b \beta \frac{d U_{i}}{d b}+c \gamma \frac{d U_{i}}{d c}=\left(a^{2}+b^{2}+c^{2}\right)^{-2 i-\frac{1}{2}}\left(a \alpha \frac{d Q_{i}}{d a}+b \beta \frac{d Q_{i}}{d b}+c \gamma \frac{d Q_{i}}{d c}\right) \\
& -(4 i+1)\left(a^{2}+b^{2}+c^{2}\right)^{-2 i-\frac{3}{2}}\left(a^{2} \alpha+b^{2} \beta+c^{2} \gamma\right) Q_{i}, \\
& \alpha^{2} \frac{d U_{i}}{d \alpha}+\beta^{2} \frac{d U_{i}}{d \beta}+\gamma^{2} \frac{d U_{i}}{d \gamma}+\frac{1}{2}(\alpha+\beta+\gamma) U_{i} \\
& =\left(a^{2}+b^{2}+c^{2}\right)^{-2 i-\frac{1}{2}}\left(\alpha^{2} \frac{d Q_{i}}{d \alpha}+\beta^{2} \frac{d Q_{i}}{d \beta}+\gamma^{2} \frac{d Q_{i}}{d \gamma}\right)+\frac{1}{2}(\alpha+\beta+\gamma) Q_{i} .
\end{aligned}
$$

Hence, putting in like manner

$$
\begin{array}{r}
(i+1)(2 i+5) Q_{i+1}=-\left(2 i+\frac{3}{2}\right)\left\{\left(a^{2}+b^{2}+c^{2}\right)\left(a \alpha \frac{d Q_{i}}{d a}+b \beta \frac{d Q_{i}}{d b}+c \gamma \frac{d Q_{i}}{d c}\right)\right. \\
\left.-(4 i+1)\left(a^{2} \alpha+b^{2} \beta+c^{2} \gamma\right) Q_{i}\right\} \\
-(2 i+1)\left(a^{2}+b^{2}+c^{2}\right)\left(\alpha^{2} \frac{d Q_{i}}{d \alpha}+\beta^{2} \frac{d Q_{i}}{d \beta}+\gamma^{2} \frac{d Q_{i}}{d \gamma}+\frac{1}{2}(\alpha+\beta+\gamma) Q_{i}\right),
\end{array}
$$

i.e.

$$
\begin{gathered}
(i+1)(2 i+5) Q_{i+1}=-\left(a^{2}+b^{2}+c^{2}\right)\left\{\left(2 i+\frac{3}{2}\right)\left(a \alpha \frac{d Q_{i}}{d a}+d \beta \frac{d Q_{i}}{d b}+c \gamma \frac{d Q_{i}}{d c}\right)\right. \\
\left.+(2 i+1)\left(\alpha^{2} \frac{d Q_{i}}{d \alpha}+\beta^{2} \frac{d Q_{i}}{d \beta}+\gamma^{2} \frac{d Q_{i}}{d \gamma}+\frac{1}{2}(\alpha+\beta+\gamma) Q_{i}\right)\right\}+\left(2 i+\frac{3}{2}\right)(4 i+1)\left(a^{2} \alpha+b^{2} \beta+c^{2} \gamma\right) Q_{i},
\end{gathered}
$$

from which the functions $Q_{i}$ may be calculated successively.

We may, it is clear, write

$$
U_{i}=\frac{4 \pi}{3} \cdot \frac{1}{2^{i} 1.2 .3 \ldots i 5.7 \ldots 2 i+3} K_{i}
$$

and we shall then have

where

$$
\begin{aligned}
\left(a^{2}+b^{2}+c^{2}\right) K_{i+1} & +(4 i+3)\left(a \alpha \frac{d K_{i}}{d a}+b \beta \frac{d K_{i}}{d b}+c \gamma \frac{d K_{i}}{d c}\right) \\
& +(4 i+2)\left(\alpha^{2} \frac{d K_{i}}{d \alpha}+\beta^{2} \frac{d K_{i}}{d \beta}+\gamma^{2} \frac{d K_{i}}{d \gamma}\right) \\
& +(2 i+1)(\alpha+\beta+\gamma) K_{i}=0
\end{aligned}
$$

$$
K_{0}=\frac{1}{\sqrt{\left(a^{2}+b^{2}+c^{2}\right)}} .
$$

I proceed to show that

$$
K_{i}=\left(\alpha \frac{d^{2}}{d a^{2}}+\beta \frac{d^{2}}{d b^{2}}+\gamma \frac{d^{2}}{d c^{2}}\right)^{i} \frac{1}{\sqrt{ }\left(a^{2}+b^{2}+c^{2}\right)} .
$$


In fact, assuming this equation for any particular value of $i$, we find first

$$
\begin{aligned}
\left(\alpha^{2} \frac{d}{d \alpha}+\beta^{2} \frac{d}{d \beta}+\gamma^{2} \frac{d}{d \gamma}\right) K_{i} & =i\left(\alpha \frac{d^{2}}{d a^{2}}+\beta \frac{d^{2}}{d b^{2}}+\gamma \frac{d^{2}}{d c^{2}}\right)^{i-1}\left(\alpha^{2} \frac{d^{2}}{d a^{2}}+\beta^{2} \frac{d^{2}}{d b^{2}}+\gamma^{2} \frac{d^{2}}{d c^{2}}\right) \frac{1}{\sqrt{\left(a^{2}+b^{2}+c^{2}\right)}} \\
& =i\left(\alpha^{2} \frac{d^{2}}{d a^{2}}+\beta^{2} \frac{d^{2}}{d b^{2}}+\gamma^{2} \frac{d^{2}}{d c^{2}}\right) \quad\left(\alpha \frac{d^{2}}{d a^{2}}+\beta \frac{d^{2}}{d b^{2}}+\gamma \frac{d^{2}}{d c^{2}}\right)^{i-1} \frac{1}{\sqrt{\left(a^{2}+b^{2}+c^{2}\right)}} \\
& =i\left(\alpha^{2} \frac{d^{2}}{d a^{2}}+\beta^{2} \frac{d^{2}}{d b^{2}}+\gamma^{2} \frac{d^{2}}{d c^{2}}\right) K_{i-1} .
\end{aligned}
$$

Now putting for shortness

$$
\Delta=\alpha \frac{d^{2}}{d a^{2}}+\beta \frac{d^{2}}{d b^{2}}+\gamma \frac{d^{2}}{d c^{2}}
$$

.we find, replacing $\Delta K_{i+1}$ and $\Delta K_{i}$ by their values $K_{i+2}$ and $K_{i+1}$,

$$
\begin{array}{lll}
\Delta\left(a^{2}+b^{2}+c^{2}\right) K_{i+1}= & \left(a^{2}+b^{2}+c^{2}\right) K_{i+2} \\
+ & +\left(a \alpha \frac{d}{d a}+b \beta \frac{d}{d b}+c \gamma \frac{d}{d c}\right) K_{i+1} \\
+ & 2(\alpha+\beta+\gamma) K_{i+1}, \\
\Delta\left(a \alpha \frac{d}{d a}+b \beta \frac{d}{d b}+c \gamma \frac{d}{d c}\right) K_{i}= & \left(a \alpha \frac{d}{d a}+d \beta \frac{d}{d b}+c \gamma \frac{d}{d c}\right) K_{i+1} \\
+2\left(\alpha^{2} \frac{d^{2}}{d a^{2}}+\beta^{2} \frac{d^{2}}{d b^{2}}+\gamma^{2} \frac{d^{2}}{d c^{2}}\right) K_{i}, & \left(\alpha^{2} \frac{d}{d \alpha}+\beta^{2} \frac{d}{d \beta}+\gamma^{2} \frac{d}{d \gamma}\right) K_{i+1} \\
\Delta\left(\alpha^{2} \frac{d}{d \alpha}+\beta^{2} \frac{d}{d \beta}+\gamma^{2} \frac{d}{d \gamma}\right) K_{i}=\quad & \left(\alpha^{2} \frac{d^{2}}{d a^{2}}+\beta^{2} \frac{d^{3}}{d b^{2}}+\gamma^{2} \frac{d^{2}}{d c^{2}}\right) K_{i}, \\
\Delta(\alpha+\beta+\gamma) K_{i}=\quad\left(\alpha+\beta+\gamma \quad K_{i+1} ;\right.
\end{array}
$$

hence operating on the equation of differences with the symbol $\Delta$, we obtain

$$
\begin{aligned}
\left(a^{2}+b^{2}+c^{2}\right) K_{i+2} & +(4 i+7)\left(a \alpha \frac{d}{d a}+b \beta \frac{d}{d b}+c \gamma \frac{d}{d c}\right) K_{i+1} \\
& +(4 i+2)\left(a^{2} \frac{d}{d \alpha}+\beta^{2} \frac{d}{d \beta}+\gamma^{2} \frac{d}{d \gamma}\right) K_{i+1} \\
+\{2(4 i+3) & -(4 i+2)\}\left(\alpha^{2} \frac{d^{2}}{d a^{2}}+\beta^{2} \frac{d^{2}}{d b^{2}}+\gamma^{2} \frac{d^{2}}{d c^{2}}\right) K_{i} \\
+ & +(2 i+3)(\alpha+\beta+\gamma \quad) K_{i+1}=0,
\end{aligned}
$$


the third line of which is

$$
4(i+1)\left(\alpha^{2} \frac{d^{2}}{d a^{2}}+\beta^{2} \frac{d^{2}}{d b^{2}}+\gamma^{2} \frac{d^{2}}{d c^{2}}\right) K_{i},=4\left(\alpha^{2} \frac{d}{d \alpha}+\beta^{2} \frac{d}{d \beta}+\gamma^{2} \frac{d}{d \gamma}\right) K_{i+1},
$$

by a foregoing equation, and the assumed equation of difference thus leads to

$$
\begin{aligned}
\left(a^{2}+b^{2}+c^{2}\right) K_{i+2} & +(4 i+7)\left(a \alpha \frac{d}{d a}+b \beta \frac{d}{d b}+c \gamma \frac{d}{d c}\right) K_{i+1} \\
& +(4 i+6)\left(\alpha^{2} \frac{d}{d \alpha}+\beta^{2} \frac{d}{d \beta}+\gamma^{2} \frac{d}{d \gamma}\right) K_{i+1} \\
& +(2 i+3)\left(\alpha+\beta+\gamma \quad \alpha_{i+1}=0\right.
\end{aligned}
$$

which is the assumed equation, writing $i+1$ instead of $i$. The equation, if true for $i$, is therefore true for $i+1$, and it is easily seen to be true for $i=0$; hence it is true generally, or the value

$$
K_{i}=\left(\alpha \frac{d^{2}}{d a^{2}}+\beta \frac{d^{2}}{d b^{2}}+\gamma \frac{d^{2}}{d c^{2}}\right)^{i} \frac{1}{\sqrt{\left(a^{2}+b^{2}+c^{2}\right)}}
$$

satisfies the equation obtained by Laplace's method, and gives, moreover, the proper value for $K_{0}$. We have thus the value of $K_{i}$; and remembering that

$$
V=\sqrt{(\alpha+\theta)(\beta+\theta)(\gamma+\theta)} v,
$$

and observing that the symbol $\Delta$ may be replaced by

$$
\Delta=(\alpha+\theta) \frac{d^{2}}{d a^{2}}+(\beta+\theta) \frac{d^{2}}{d b^{2}}+(\gamma+\theta) \frac{d^{2}}{d c^{2}}
$$

the value of $V$ is

$$
V=\frac{4 \pi}{3} \sqrt{ }(\alpha+\theta)(\beta+\theta)(\gamma+\theta) S_{i}^{\infty}\left(\frac{1}{2^{i} 1.2 \ldots i .5 .7 \ldots 2 i+3} \Delta^{i} \frac{1}{\sqrt{\left(a^{2}+b^{2}+c^{2}\right)}}\right)
$$

which is in fact the value which I have found by a much more simple method in the Cambridge Mathematical Journal, t. III. p. 69 [2]. 